\title{
NOVEDADES EN LA AMAZONÍA PERUANA
}

\author{
Luis D. Gómez P. \\ Organización para Estudios Tropicales, apdo. 676-2050, San Pedro, Costa Rica. \\ Academia Nacional de Ciencias \\ Botanical Research Institute of Texas \\ gomezp@ots.ac.cr
}

\begin{abstract}
Aвstract. The aquatic plant Phyllanthus fluitans Benth. ex Müll. Arg. (Phyllanthaceae) is reported from the Ucayali, Napo, and Amazon rivers. An unusual form of Anetium citrifolium (L.) Splitg. (Vittariaceae) is here described as a new variety.
\end{abstract}

Resumen. La planta acuática Phyllanthus fluitans Benth. ex Müll. Arg. (Phyllanthaceae) se comunica para la cuenca amazónica del Perú en los ríos Ucayali, Napo, Amazonas y afluentes. Una forma divergente de la típica se describe como una nueva variedad de Anetium citrifolium (L.) Splitg. (Vittariaceae).

Palabras clave / Key words: Perú, Peruvian Amazon, Phyllanthus fluitans, Anetium citrifolium.

La familia Euphorbiaceae s.l. contiene más de 6000 especies en unos 350 géneros, de las cuales dos son hidrófilas, una anfibia, Caperonia palustris (L.) St.-Hill., de amplia distribución, y una flotante, Phyllanthus fluitans Benth. ex Müll. Arg. (Linnaea 22: 36.1863, Phyllanthaceae Martynov), conocida de Argentina, Bolivia, Brasil, Ecuador, México, Paraguay y Venezuela (Chodat 1908, Cook 1974, Cowan 1983, Jørgensen \& León-Yánez 1999). Esta especie generalmente está representada en los herbarios por pocos ejemplares, no tanto por su rareza sino porque su reducido tamaño la hace casi imperceptible en las alfombras de plantas acuáticas flotantes asociadas, tales como Azolla filiculoides, Salvinia spp., Pistia stratiotes, Lemna spp., etc.

En su tratamiento de la familia para la flora peruana, McBride (1951) sugiere su presencia en ese país tan rico en ambientes lóticos y lénticos, particularmente en la cuenca amazónica. Braco \& Zarucchi (1993) también la incluyen, fide McBride, en el catálogo de plantas peruanas. Sin embargo, no aparece citada en Vásquez (1997) para ninguna de las reservas biológicas de Iquitos. Durante el curso Ecología de Ecosistemas Amazónicos de 2001 y 2002, en compañía de R. Vásquez, la recolectamos en la Cocha Shimigai en la margen derecha del río Napo. En mi recorrido de los ríos Ampayacu y Oroza en 2003 también tuve oportunidad de observarla y hacer un ejemplar. En 2005 localicé una población considerable en la cabecera del río Zapote, de aguas negras, afluente del Canal de Puinahua, entre los ríos Ucayali y Marañón. Estos hallazgos se comunican aquí (Fig.1).
EsPeCímenes. Perú: Cocha Shimigai, 2 km aguas arriba de la desembocadura del Sucusari, margen derecha del Napo, Loreto, Gómez \& Vásquez s.n. (AMAZ); Cocha, $3 \mathrm{~km}$ al sur de Estación Biológica Madreselva, río Oroza, Dep. Amazonas, Gómez 25436 (AMAZ); Cabeceras de río Zapote, Canal de Puinahua, Reserva Pacaya-Samiria, Loreto, Gómez 26237 (AMAZ). Reserva Pacaya-Samiria, McDaniel \& Rimachi 22388 (MO).

Otros ejemplares estudiados. Argentina: Chaco, Meyer T 16290 (MO); Formosa, Guaglianone \& Múlgura 2299 (SI). Bolivia: Santa Cruz, Montana, A. Fuentes 583 (USZ). Brasil: Rio de Janeiro, A. Gentry 49739 (MO). Ecuador: Guayas, Holm-Nielsen \& Jeppesens 71 y 7223 (AAU); Cuyabeno, L.D. Gómez 25993, (USJ). México: Tabasco, Cowan \& Amador 2736 (MO), Cowan 2825 (MO). Paraguay: Neembuco, Meyer T 16168 (C, LIL, S). Venezuela: Colonnelo 1612 (MO).

El género Anetium Splitg. (Tijd. Natuurl. Gesch. Physiol. 7: 395. 1840, basado en Acrostichum citrifolium L., Sp. Pl. 2: 1067. 1753; Pteridanetium Copeland, Gen. Filicum 224. 1947, nom. superfl.) es un pteridófito de la familia Vittariaceae considerado monoespecífico y se encuentra distribuido desde México hasta Bolivia y en las Antillas, excepto en Cuba. La especie Anetium citrifolium (L.) Splitg. (loc. cit.) es muy homogénea en su morfología en toda su área de distribución. Sin embargo, durante mis viajes a diversas regiones de la cuenca amazónica peruana he observado poblaciones muy divergentes de las 
formas conocidas. Estas diferencias fueron notadas por Hooker en ejemplares recogidos por Richard Spruce [Amazonas, Spruce 2368, y Pará, Brasil, Spruce 32 (E)] y nombrados Antrophyum sprucei, in scheda y sin descripción. Este nombre fue tomado por T. Moore y publicado como Anetium sprucei Moore (Ind. Fil. 72: 1857 y 73: 1858), también sin descripción alguna.

Al respecto dice Hooker: "Mr. Moore has entered this under Anetium as a distinct species, and has taken the name (not without acknowledgement) from my private herbarium, but without careful examination or any description," como nota al pie de la página donde incluye lo que obviamente consideró una variedad de su concepto Hemionitis (Anetium) citrifolia (Species Filicum 5: 193. 1864), como sinónimo. Sin embargo, la acuciosa observación de Hooker fue precedida unos años antes por Fée en su Mém. Fam. Fougères. 3-4 Histoire des Antrophyées 4: 52. 1851-1852, con ejemplares recogidos por Leprieur en la Guyana Francesa. Cito textualmente:

"Antrophyum pendulum Leprieur, inéd.

Anetium citrifolium, F., Hist. des Acrostichées p. 97: 1852 var $\beta$ flaccidum.

Guyanne par Leprieur 1830, 1839.

40-50 cm de longeur sur 7-9 mill de largeur

Cette grande espèce que nous avions établie comme une varieté de l'Anetium citrifolium avec l'épithète de flaccidum, mieux connue, semble devoir constituer une espèce. Elle parait être jusq'à présent particuliér à la Guyanne où elle se plait sur les rives des fleuves. M. Lepreur 1'a recuille sur le stipe des 'manicaria'. Elle y est pendentè et sa consistence est flasque, même á l'état vivant.

Elle differe de 1'A. citrifolium par une consistence molle, un marge flexeuse, des dimension plus considérables, un port different et des écailles dont la marge simplement dentée n'est pas frangée; desséchée, elle perde l'elasticité que conserve dans les herbiers $l ' A$. citrifolium. Il faut, au reste, que de nouvelles observations viennent confirmer la validité de cette espèce."

A la excelente descripción de Fée puedo agregar que estas plantas presentan láminas de hasta 110 $\mathrm{cm}$ o más de longitud y $15 \mathrm{~cm}$ de ancho en su parte media, que la costa, inmersa, llega casi hasta el ápice. Las láminas son distantes $5-14 \mathrm{~cm}$. Los rizomas son largamente reptantes, de hasta $1 \mathrm{~cm}$ de diámetro, glabros, algo prismáticos como lo son los estípites, muy desarrollados, de hasta $10 \mathrm{~cm}$ de longitud. El ápice rizomático presenta unas pocas escamas clatradas, caedizas. A diferencia de las formas comunes, el rizoma no presenta densidad de raicillas afelpadas en toda su longitud, sino que éstas son, en cambio, fibrosas, pocas y largas, no afelpadas sino rígidas, escleróticas, y se producen mayoritariamente opuestas a los estípites.

En las formas típicas las láminas rara vez sobrepasan $40 \mathrm{~cm}$ de largo y $5 \mathrm{~cm}$ de ancho en el medio, son de textura herbácea, oval-lanceoladas, de ápice subagudo. La costa no sobrepasa los dos tercios de la longitud de la fronde. Las hojas están próximas, casi fasciculadas. Rizoma densamente provisto de raicillas afelpadas que se adhieren al sustrato tenazmente. Ápice del rizoma densamente palmáceo. Escamas clatradas, cuasifimbriadas, iridiscentes.

Si en la Guyana Leprieur la encontró sobre estípites de palmas del género Manicaria, en la Amazonía peruana sólo la he observado en palmas de toca (Attalea tessmannii Burret), cerca de cursos de agua o en selva inundable, formando masivas cepas y no, como sucede con $A$. citrifolium típico, plantas por lo general aisladas. Por estas razones, considero válido revalidar el epíteto de Fée como:

\section{Anetium citrifolium (L.) Splitg. var. pendulum (Leprieur in Fée) L.D. Gómez, var. nova}

Laminae usque ad 112-plure longae, $12-15 \mathrm{~cm}$ latae, coriaceae, elongatae, undulatae, praesertim stipitatae. Rhizomate glabro, paucissime radicato, apicem paucissime squamato, squamis clathratis.

\section{Holotypus (hic designatur): Leprieur 1830 (P).}

Paratipos. Perú: Paucarillo, río Oroza, Amazonas, Loreto. L.D. Gómez 26207 (AMAZ); Madre Selva, cerca de aldea de Comandancia, Amazonas, Loreto, 125 m. L.D. Gómez 26212 (CR); bosque secundario, margen izquierda río Zapote, sobre "toca", Canal de Puinahua del Ucayali, Loreto. L.D. Gómez 26238 (USJ).

Un estudio de las isozimas de estas plantas podría establecer, más allá de la duda, la monotipia del género Anetium.

Agradecimientos. A los estudiantes del curso Ecología de los Ecosistemas Amazónicos, que me acompañaron 
en el campo; a la Universidad de la Amazonía Peruana, las autoridades de INRENA por la autorización (084Iquitos, a la Organización para Estudios Tropicales. A 2004 a BRIT) para recolectar flora en el Perú.

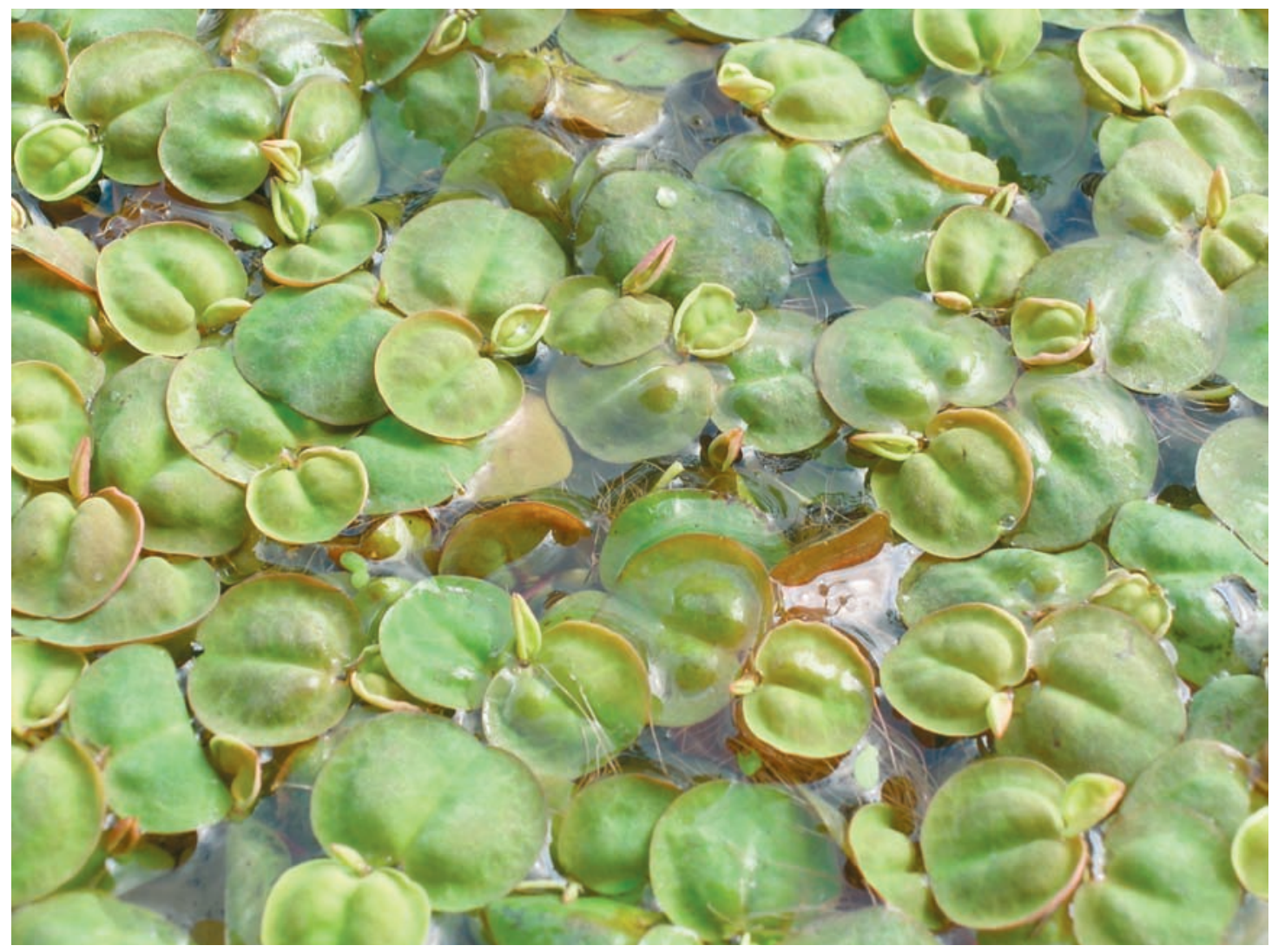

Fig. 1. Phyllanthus fluitans, el diámetro promedio de las hojas: $20 \mathrm{~mm}$. Cocha, Río Zapote, Canal de Puinahua, Reserva Pacaya-Samiria, Loreto, Perú. (Foto: L. D. Gómez)

\section{LITERATURA CITADA}

Braco, L. \& J.L. Zarucchi. 1993. Catalogue of the flowering plants and conifers of Perú. Monogr. Syst. Bot. Missouri Bot. Gard. 45: 1-1285.

Chodat, R. 1906. Observations sur le macroplancton des étangs du Paraguay. Bull. Herb. Boissier, sér.2, 6: 143-147.

Cook, C.D.K. 1974. Water plants of the world. Junk, La Haya, p. 239-241.

Cowan, C.P. 1983. Flora de Tabasco. Listados florísticos de México 1: 1-123.

Jørgensen, P. \& S. León-Yánez. 1999. Catalogue of the vascular plants of Ecuador. Monogr. Syst. Bot. Missouri Bot. Gard. 75: 1-1182.

McBride, J.F. 1951. Euphorbiaceae. Flora of Perú. Field Mus. Nat. Hist. Bot. Ser. 13(3A/1): 3-200.

Vásquez M., R. 1997. Flórula de las Reservas Biológicas de Iquitos, Perú. Monogr. Syst. Bot. Missouri Bot. Gard. 63:1-1046. 
